\title{
Efectos macroeconómicos de la inmigración. Impacto sobre el empleo y los salarios de los nativos
}

Amparo González Ferrer

CEACS, Instituto Juan March

agonzalez@ceacs.march.es

\section{Resumen}

El presente artículo examina la reflexión teórica y la evidencia empírica disponible acerca de los efectos macro-económicos de la mano de obra inmigrante. En particular sus efectos sobre el empleo y los salarios de los autóctonos. Tras desentrañar las asunciones subyacentes a la hipótesis del reemplazo y de la segmentación, compara los resultados de los estudios empíricos realizados en EEUU y en Europa. A la luz de dicha evidencia concluye que la inmigración no ha provocado un aumento del desempleo, ni en EEUU ni en Europa, si bien puede haber contribuido, aunque menos que la apertura comercial, al incremento de la desigualdad salarial en EEUU desde los años ochenta.

Palabras clave: inmigración, desempleo, desigualdad salarial, sustitución, complementariedad.

\section{Abstract}

This article analyses both the literature and available evidence on the macro-economic effects of immigration. In particular, it focuses on its effects on the native employment and wages. After disentangling the assumptions underlying the substitution and segmentation hypotheses, it compares the empirical results obtained by the European and American investigations. From this evidence, it concludes that immigration has not increased the native unemployment, neither in USA nor Europe, but it might have contributed - although in a minor extent than international trade openness - to the increasing wage inequality in USA since the early eighties.

Key words: immigration, unemployment, wage inequality, substitution, complementariety.

* Agradezco a Aron Cohen y J. Rafael Morillas que me animaran a escribir este artículo, y a José Ma Maravall, Javier Ramos y José Fernández sus valiosas críticas y comentarios. 


\section{Sumario}

$\begin{aligned} \text { 1. Introducción } & \text { 3. Evidencia empírica. } \\ \text { 2. Marco teórico. La hipótesis } & \text { EEUU versus Europa } \\ \text { del desplazamiento y la hipótesis } & \text { 4. Conclusiones } \\ \text { de la segmentación } & \text { Bibliografía }\end{aligned}$

\section{Introducción}

El análisis económico de la inmigración (término éste, inmigración, que ya indica el punto de vista adoptado, el de los países receptores) adquirió una creciente relevancia en el debate político de EEUU a principios de los ochenta, debido no sólo al elevado volumen de entradas de trabajadores extranjeros durante la década anterior, sino también a las transformaciones que el mercado de trabajo norteamericano comenzaba a experimentar por entonces: deterioro de los salarios, creciente desigualdad, declive sindical, creciente pobreza infantil, etc. En Europa, sin embargo, el fin de los programas de reclutamiento de mano de obra extranjera por parte de los países centroeuropeos a mediados de los setenta, dejaba aparentemente sin razón de ser un análisis detallado del impacto de la inmigración sobre una economía en recesión. En principio, el retorno de los trabajadores extranjeros y el cierre de fronteras significaría el fin de la cuestión. De hecho, sólo años más tarde, una vez se hubo tomado conciencia de que, pese al giro político, la entrada de extranjeros con la intención de trabajar seguía siendo una realidad, la investigación sobre la materia recibió un nuevo impulso.

Este trabajo pretende proporcionar una visión sintética del estado de la investigación relativa al impacto económico de la inmigración sobre el mercado de trabajo de los países receptores, concretamente sobre el nivel de empleo y los salarios, detectar algunas de las debilidades teóricas y metodológicas, y reflexionar sobre las divergentes conclusiones alcanzadas por las investigaciones existentes.

\section{Marco teórico. La hipótesis del desplazamiento y la hipótesis de la segmentación}

La motivación que generalmente guía el análisis económico de la inmigración es la de refutar, o por el contrario apoyar, una idea muy difundida, la de que la mano de obra inmigrante desplaza a trabajadores nacionales de sus puestos de trabajo y/o provoca una reducción de los salarios.

Ésta es una idea bastante común entre la población de los países avanzados receptores y que con frecuencia se invoca como argumento sólido para justificar una actitud de rechazo a la inmigración. Por ceñirnos al caso español, podríamos citar los resultados de las encuestas del CIRES, realizadas entre 1991 y 1995, así como alguna más reciente realizada por el IMSERSO, resumidos en la tabla 1 . 
Tabla 1. Opinión de los españoles sobre la influencia de los inmigrantes sobre el paro y los salarios.

\begin{tabular}{lccccc}
\hline & \multicolumn{2}{c}{ País subdesarrollado } & & \multicolumn{2}{c}{ País desarrollado } \\
\cline { 2 - 5 } \cline { 5 - 6 } & $\mathbf{1 9 9 2}$ & $\mathbf{1 9 9 7}$ & & $\mathbf{1 9 9 1}$ & $\mathbf{1 9 9 7}$ \\
\hline Más paro & $62 \%$ & $46 \%$ & $33 \%$ & $33 \%$ \\
No afecta & $34 \%$ & $47 \%$ & $55 \%$ & $57 \%$ \\
NS/NC & $4 \%$ & $7 \%$ & $11 \%$ & $11 \%$ \\
\hline Menos salarios & $38 \%$ & $29 \%$ & $15 \%$ & $18 \%$ \\
No afecta & $54 \%$ & $59 \%$ & $64 \%$ & $67 \%$ \\
Más salarios & $1 \%$ & $1 \%$ & $6 \%$ & $2 \%$ \\
NS/NC & $7 \%$ & $10 \%$ & $15 \%$ & $14 \%$ \\
\hline
\end{tabular}

Fuente: los datos de 1991 y 1992 pertenecen al CIRES, La realidad social en España. Los datos de 1997 pertenecen al IMSERSO.

\section{Hipótesis del desplazamiento o reemplazo}

Una forma sencilla de exponer el razonamiento económico que subyace a la "hipótesis del desplazamiento o reemplazo" es a través de un modelo de equilibrio general determinado por la concurrencia de la oferta y la demanda en el mercado de trabajo del país de acogida. Pese a sus limitaciones ${ }^{1}$, dicho modelo resulta muy útil en términos pedagógicos, pues permite conocer las condiciones necesarias para que el reemplazo se produzca, así como reflexionar sobre la verosimilitud de las mismas.

El análisis de oferta y demanda de trabajo responde al enfoque de la economía neoclásica. Partiendo de la teoría estándar del comercio internacional, se consideran los flujos de personas completamente equiparables en su tratamiento a los flujos de mercancías y, en consecuencia, un medio alternativo para lograr la equiparación de los precios de factores de producción a nivel mundial (Borjas, 1989: 459; Jahn y Straubhaar, 1998: 25; Coleman, 1999: 488). Así, en la perspectiva neoclásica, el diferencial de salarios entre países es la principal causa subyacente a las migraciones internacionales.

Analizaremos, por medio de los gráficos, los efectos de la entrada de una determinada cantidad $(I)$ de mano de obra inmigrante (gráfico 1).

Inicialmente nos encontramos con un mercado de trabajo definido por la situación de equilibrio $\left(S_{n}, E_{n}\right)$. La entrada de una cantidad (I) de nuevos trabajadores, procedentes del exterior, provoca un aumento de la mano de obra disponible en el país en cuestión, es decir, un aumento de la oferta de trabajo que representamos mediante una nueva curva de oferta $\left(O_{I}\right)$, a la derecha de la

1. Limitaciones relacionadas con la posibilidad de valorar otros elementos más allá de las diferencias salariales, como cambios en las regulaciones de entradas y salidas de personas, tanto en los países emisores como en los receptores, la existencia de motivaciones no económicas en la decisión de emigrar o la intervención en el modelo de más de dos países. 


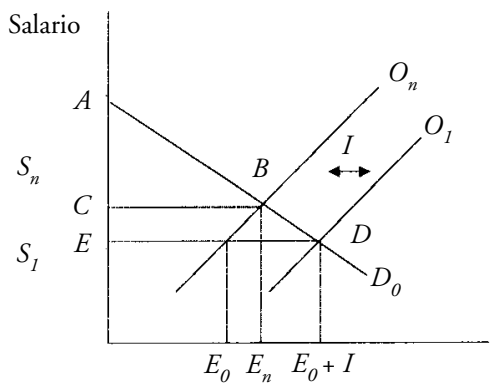

Gráfico 1. Modelo estándar.

anterior $\left(O_{n}\right)$. Este desplazamiento implica una nueva situación de equilibrio $\left(S_{P}, E_{O}+I\right)$. Comparando la situación de equilibrio inicial $\left(S_{n}, E_{n}\right)$ con la nueva $\left(S_{P}, E_{0}+I\right)$, observamos que se ha producido:

- Una reducción del salario de equilibrio (de $S_{n}$ a $S_{I}$ ).

- Una reducción en el número de trabajadores nacionales empleados (de $E_{n}$ a $\left.E_{0}\right)$.

- Un aumento de la remuneración del capital o, en general, del resto de factores de producción (del área del triángulo $A B C$ a la del triángulo $A D E$ ).

Ahora bien, no debemos pasar por alto el hecho de que al análisis realizado (paso de la situación de equilibrio inicial a la situación determinada por el incremento de la mano de obra disponible) subyacen ciertas asunciones: a) se asigna arbitrariamente una determinada pendiente a las curvas de oferta y de demanda de trabajo, es decir, una determinada sensibilidad de las mismas a las variaciones de salario; $b$ ) se mantiene fija la demanda de trabajo; $c$ ) se trata el factor trabajo, el conjunto de los trabajadores, como un factor absolutamente homogéneo, es decir, sin diferenciaciones internas de ningún tipo (cualificación, productividad, etc.) y por tanto perfectamente sustituibles entre sí; d) se permite un reajuste completo del mercado vía salarios, lo que equivale a presuponer salarios perfectamente flexibles.

Evidentemente llegaríamos a conclusiones muy distintas a las señaladas (bajada de salarios, reducción del empleo nativo) si modificásemos algunos de esos supuestos. Veamos qué ocurriría considerando:

\section{Curvas de oferta y demanda de trabajo con pendientes diferentes a las repre- sentadas.}

Si las elasticidades de ambas curvas fuesen otras, los efectos variarían sustancialmente, al menos en su magnitud. Por ejemplo, ante una curva de oferta de trabajo nativo prácticamente fija (vertical), es decir casi insensible a las variaciones de salario, la entrada de mano de obra inmigrante no tendría apenas ningún efecto sobre el nivel de empleo nativo. En cambio, cuanto más 
Tabla 2. Posibles efectos sobre el empleo y los salarios según las elasticidades.

\begin{tabular}{llll}
\hline & $\begin{array}{l}\text { Oferta (casi) } \\
\text { inelástica }\end{array}$ & $\begin{array}{l}\text { Oferta } \\
\text { poco elástica }\end{array}$ & $\begin{array}{c}\text { Oferta } \\
\text { muy elástica }\end{array}$ \\
\hline $\begin{array}{l}\text { Demanda (casi) } \\
\text { inelástica }\end{array}$ & $\begin{array}{l}\text { Empleo nativo igual } \\
\text { Brutal reducción salarial }\end{array}$ & $\begin{array}{l}\text { Reducción empleo nativo } \\
\text { Fuerte reducción salarial }\end{array}$ & $\begin{array}{c}\text { Brutal reducción } \\
\text { empleo nativo } \\
\text { Bajada salarial } \\
\text { Efecto } \\
\text { desplazamiento }\end{array}$ \\
\hline $\begin{array}{l}\text { Brutal reducción } \\
\text { salarial }\end{array}$ & & Reducción empleo nativo \\
elástica & Empleo nativo igual & $\begin{array}{c}\text { Fuerte reducción } \\
\text { empleo nativo }\end{array}$ \\
\hline $\begin{array}{l}\text { Femanda bajada salarial } \\
\text { elástica }\end{array}$ & Bajada salarial & Fuerte bajada salarial \\
\hline
\end{tabular}

sensible a las variaciones de salario sea la oferta (más plana), y menos sensible (más vertical) sea la demanda, mayor será el desplazamiento de trabajadores nacionales por no nacionales en el mercado de trabajo.

Por tanto, la entrada de mano de obra extranjera implicaría distintos efectos sobre el empleo y los salarios en el país receptor según distintas pendientes en las curvas de oferta y demanda de trabajo ${ }^{2}$, como resume la tabla 2. Los gráficos 2 y 3 representan los casos extremos de oferta inelástica y de demanda muy elástica.

\section{Posibles desplazamientos de la curva de demanda que podrían producirse a raíz} de la entrada de inmigrantes.

Por una parte, como ya señaló Harrison a comienzos de los ochenta, desde el momento de su llegada, un inmigrante realiza un consumo adicional en bienes y servicios, incluso si no empieza a trabajar de inmediato, que estimulará la producción y que, en consecuencia, puede estimular la demanda de trabajo. Para que dicho aumento de la demanda de trabajo se tradujera automáticamente en una reducción del paro, entre los nativos tendrían que darse unas condiciones poco probables (rigidez en los salarios y una tasa de desempleo global invariable). Sin embargo, lo que Harrison señaló sigue siendo válido: el que los inmigrantes asuman como parte de su proyecto migratorio un período inicial de desempleo y que, por tanto, a su llegada, el desempleo entre ellos sea mayor que el de los nativos, no tiene por qué interpretarse como una presión añadida sobre los nativos, sino que, al contrario, podría ser indicio de mayores oportunidades de trabajo.

2. No debemos olvidar, en relación con esta cuestión, la dificultad para realizar estimaciones de las respectivas elasticidades. 


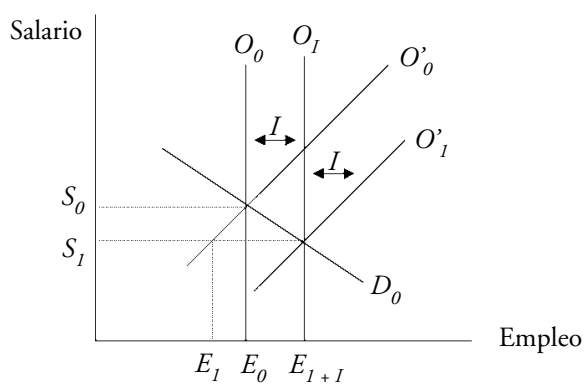

Gráfico 2. Oferta inelástica.

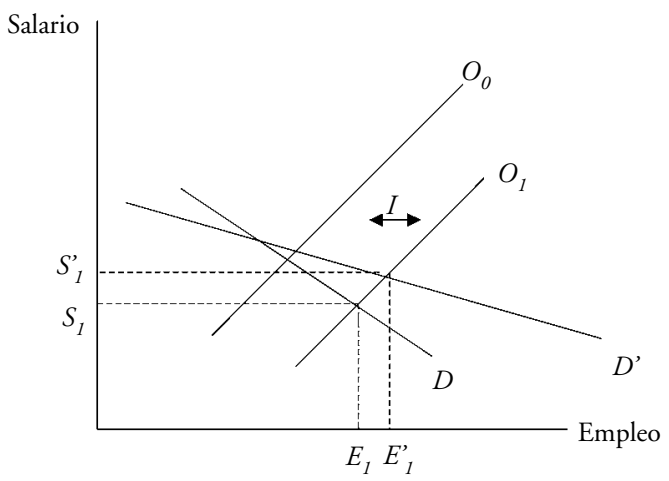

Gráfico 3. Demanda elástica.

Por otra parte, parece posible que un inmigrante induzca la creación de un nuevo puesto de trabajo, ya sea porque ofrece una serie de cualificaciones atractivas para el empresario, ya sea porque está dispuesto a desempeñar tareas por una salario inferior al habitual. Así, el proceso no es tanto que un inmigrante ocupe un puesto de trabajo ya existente, sino, más bien, que cuando un inmigrante induce la creación de un nuevo empleo, se producen una serie de cambios en la demanda de trabajo en otros lugares de la economía. Se trata, pues, de un análisis en términos de señales y expectativas, más que de puestos de trabajo conectados con el capital físico ${ }^{3}$.

3. Evidentemente habría que explicar por qué la contratación de un inmigrante generaría esa cadena beneficiosa de acontecimientos y no lo haría del mismo modo la contratación de un nativo. La distinción entre «puesto existente» $\mathrm{y}$ «nuevo puesto de trabajo» resulta algo artificial, pero tiene sentido en la medida en que consideremos que donde alguien ya estaba trabajando, la contratación de un "sustituto» no va a generar los ajustes mencionados en el resto del sistema económico, pues éstos ya se produjeron cuando el puesto se creó. 
La llegada de inmigrantes puede aumentar la duración del desempleo de los nativos que ya estaban parados, pues la "cola» de los solicitantes de empleo se alarga y, por tanto, también el tiempo de espera. Sin embargo, a medio o largo plazo, la cadena de creación de empleos descrita podría ir compensando ese alargamiento de la cola y de duración del desempleo ${ }^{4}$.

En definitiva, la convicción que subyace a la inmovilidad de la curva de demanda es a) que el número de puestos de trabajo que puede ofrecer una economía es fijo, algo que no responde a la realidad, al menos en el medio y largo plazo, o b) que la tasa de inmigración es tan baja que los efectos adicionales sobre la demanda agregada son despreciables. Si éste último es el caso, sería lógico preguntarse por qué entonces una inmigración laboral tan reducida sí que iba a tener efectos sobre los salarios o el desempleo de los nativos. En cambio, si se produjeran desplazamientos inducidos de la demanda agregada, el potencial efecto negativo sobre los salarios podría paliarse o incluso invertirse ${ }^{5}$.

\section{El factor trabajo como un factor no homogéneo.}

En el estadio de desarrollo actual, la especialización y división del trabajo se basa sobre todo en el grado de cualificación de la mano de obra, que establece a menudo serias fronteras entre distintas categorías de trabajadores. Tales fronteras significan que los trabajadores no son perfectamente intercambiables entre sí y que, por tanto, no podemos hablar genéricamente de un mercado de trabajo en el que se observe una competencia entre todos los trabajadores por los puestos disponibles, sino de un mercado segmentado o dual ${ }^{6}$. Debemos resaltar, pues, que la consideración de distintas clases de trabajadores permitiría centrar el análisis en los efectos de la mano de obra inmigrante sobre los trabajadores nativos cualificados o poco cualificados, según sea razonable en función de las características de los inmigrantes que esté recibiendo el país. La hipótesis de la segmentación será analizada en el apartado B.

\section{Algunas rigideces institucionales en el mercado de trabajo.}

$\mathrm{Si}$, por ejemplo, en el mercado receptor existe un salario mínimo que impide la caída de los salarios por debajo de él, los reajustes necesarios tras el incre-

4. La consideración de elementos institucionales, como la generosidad del subsidio de desempleo, condicionarían de modo sustancial los efectos del proceso descrito. Por ejemplo, algunos trabajos realizados para el caso español (Bover et al., 1997), señalan que la recepción del subsidio de desempleo reduce significativamente la probabilidad de abandonar el desempleo. En cualquier caso, esta cuestión excede el ámbito de este trabajo.

5. De todos modos, aunque teóricamente es posible que el desplazamiento de la demanda, inducido por la inmigración, domine el desplazamiento de la oferta, anulando cualquier reemplazo o bajada de salarios, no parece que éste sea el caso en la economía norteamericana contemporánea (Splenger, 1956; Kelley, 1972).

6. No se trata de términos plenamente sinónimos. El mercado dual supone una simplificación de la idea de mercado segmentado. En cualquier caso, en ambos subyace la idea de que el mercado de trabajo no es un fenómeno continuo sino que contiene diferentes segmentos: dos (primario y secundario) cuando hablamos de mercado dual, más de dos si genéricamente nos referimos a mercado segmentado. 


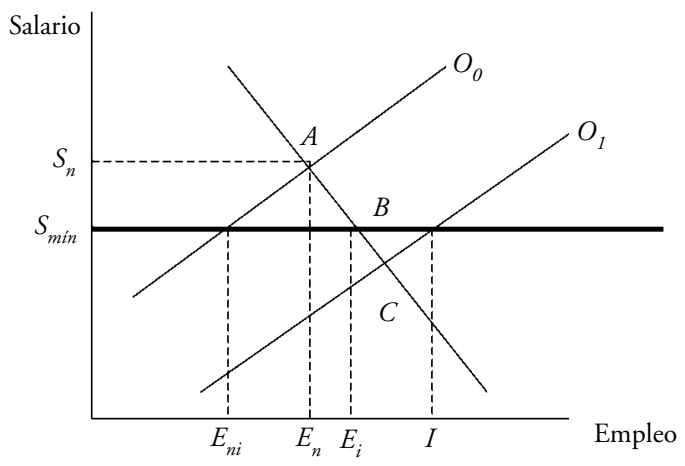

Gráfico 4. Salario mínimo.

mento de la oferta de trabajo provocado por la llegada de inmigrantes no podrán producirse únicamente vía salarios (gráfico 4 ).

Partimos de una situación en el mercado de trabajo nacional en la que el salario de equilibrio es superior al salario mínimo (punto A). La entrada de mano de obra adicional provocará: a) un descenso del salario de equilibrio, limitado al nivel del salario mínimo; b) un aumento del desempleo entre la mano de obra nacional (de $E_{n}$ a $E_{n i}$ ) porque algunos trabajadores ya no están dispuestos a trabajar por el nuevo salario; $c$ ) la contratación de una fracción de la mano de obra no nacional (de $E_{n i}$ a $\left.E_{i}\right)$, y d) el desempleo de algunos trabajadores no nacionales (de $E_{i}$ a $I$ ) que, aún estando dispuestos a trabajar por un salario inferior al salario mínimo, no encuentran trabajo. Es evidente que este último grupo de trabajadores representará un estímulo en potencia para el desarrollo de actividades de economía sumergida, en las que los empleadores puedan eludir las rigideces institucionales. Por otra parte, el tiempo que permanezcan en el paro los trabajadores nativos que quedan desempleados tras la reducción del nivel salarial, dependerá de muchos factores: la cuantía y duración de las protecciones por desempleo, el efecto dinamizador que tenga sobre el mercado el aumento del empleo total que se ha producido, las posibilidades y la disposición de dichos parados para reciclar sus cualificaciones, etc.

En resumen, si los salarios son rígidos a la baja (y no perfectamente flexibles como suponíamos en el gráfico inicial), el mayor coste que hay que soportar es un aumento del desempleo global, tanto de nativos como de inmigrantes. Ésta es la gran diferencia que se observa cuando, en lugar de mercados perfectamente competitivos, estamos ante economías con un salario mínimo.

En este punto de la exposición, es importante señalar la escasez de trabajos que analicen hasta qué punto las conclusiones sobre el impacto económico de la inmigración, derivadas del modelo estándar de mercados perfectamente competitivos, resultarían modificadas al considerar la existencia de constricciones institucionales, tan habituales en los mercados de trabajo europeos. La 
razón puede ser que la mayor parte de la investigación procede de EEUU, donde el funcionamiento del mercado de trabajo parece estar más próximo al modelo competitivo; quizás por este motivo, entre otros que mencionaremos una vez revisada la evidencia empírica a uno y otro lado del Atlántico, las conclusiones para América del Norte no sean fácilmente extrapolables al caso europeo (véase epígrafe 3).

\section{Diferente lapso temporal.}

Los efectos hasta ahora mencionados dependen de que estemos considerando el corto o el largo plazo. Mientras que en el corto plazo lo interesante sería estudiar los grados de complementariedad o sustituibilidad entre los diferentes factores productivos (capital, trabajo nativo e inmigrantes), pues los rendimientos de los complementarios aumentarían y los de los sustitutos descenderían, en el largo plazo sería necesario tomar en consideración otros elementos que pueden mediatizar los resultados. Por ejemplo, en el largo plazo el capital ya no se considera fijo y los ajustes de capacidad productiva pueden hacerse a través de él, no sólo de la mano de obra, y, por otra parte, la mano de obra inmigrante puede haber realizado inversiones en capital humano que modifiquen sus relaciones de complementariedad y sustitución con el resto de factores. Dicho de otro modo, los grupos de trabajadores nativos para los que, en un primer momento, los inmigrantes eran potenciales competidores (si es que los hay) pueden diferir de los potenciales perjudicados en momentos posteriores.

\section{Hipótesis de la segmentación}

La hipótesis de la dualización o segmentación del mercado de trabajo emerge frente al enfoque neoclásico. Desde mediados de los setenta, diferentes autores (Abrams y Abrams, 1975; Piore, 1979) ponen en cuestión no ya la magnitud de los efectos que la economía neoclásica atribuía a la recepción de mano de obra extranjera, sino la propia existencia de tales efectos.

Este enfoque hace hincapié en los denominados "factores de atracción» (pull factors), frente a los «factores de expulsión» (push factors), a la hora de explicar los movimientos migratorios: son características estructurales de las economías de los países ricos, en especial la dificultad para aumentar los salarios de los trabajadores peor pagados, las que generan una demanda continua de trabajadores dispuestos a desarrollar tareas desprestigiadas y mal pagadas. Por tanto, los inmigrantes extranjeros no obtienen puestos de trabajo a expensas o en detrimento del empleo de los nacionales, por la simple razón de que el mercado de trabajo de las economías avanzadas está lo suficientemente segmentado (diferenciado en función de distintos criterios) ${ }^{7}$ como para preser-

7. El criterio decisivo en virtud del cual el mercado de trabajo queda internamente diferenciado hasta el extremo de la segmentación, no está claramente establecido en la literatura. Estabilidad, salarios, cualificación..., han sido mencionadas como variables divisorias entre 
var a los trabajadores nacionales de un efecto directo del empleo de trabajadores extranjeros. Es más, con frecuencia esa demanda de mano de obra extranjera se retroalimenta a medida que tales tareas cada vez quedan más identificadas con "tareas propias de inmigrantes» y la segmentación deviene permanente.

Antes de apresurarnos y sustituir el análisis neoclásico por la hipótesis de la segmentación, que sin duda no carece de cierta lógica, debemos desentrañar las asunciones implícitas en ésta última:

1. En primer lugar, para ser verificada en su formulación extrema, la hipótesis de la segmentación requeriría una curva de demanda de trabajo absolutamente sensible a las variaciones de salario, es decir, horizontal. Esto equivale a suponer, entre otras cosas, una perfecta sustitución de los factores en el proceso productivo que no existe ni en el largo ni, menos aún, en el corto plazo.

2. En segundo lugar, esa idea de una demanda continua de inmigrantes dispuestos a aceptar «malos trabajos» (en términos de condiciones de trabajo, peligrosidad, número de horas, etc.) con bajos salarios, asume un mercado de trabajo con unas rigideces extremas, incapaz de modificar la jerarquía salarial o los niveles de protección laboral.

En cualquier caso, merece la pena reflexionar sobre la posibilidad de que la segmentación exista realmente en determinados sectores productivos o sobre la posibilidad de una segmentación parcial, es decir, la combinación de noefecto sobre el empleo pero sí sobre los salarios o viceversa, en función de la elasticidad o inelasticidad de la oferta de mano de obra doméstica.

\section{Evidencia empírica. EEUU versus Europa}

Uno de los fenómenos que más atención ha recibido desde principios de los ochenta en el ámbito de los países desarrollados, ha sido la diferente evolución experimentada por el empleo y los salarios en EEUU y Europa ${ }^{8}$. Mientras que el principal problema al que tuvieron (y tienen) que hacer frente los gobiernos europeos es el del aumento y la persistencia del desempleo, especialmente entre algunos sectores de su población, EEUU vio tambalearse uno de los cimientos del «sueño americano»: el trabajo duro ya no aseguraba un trozo en el reparto del pastel. Pese a la elevada productividad y los altos estándares de vida que, en media, seguía ofreciendo su economía, EEUU había dejado de ser uno de los líderes mundiales en la oferta de buenos trabajos con buenos salarios.

«buenos» $\mathrm{y}$ «malos» trabajos. Sin embargo, las clasificaciones que cada criterio suministraría son diferentes. Quizá el único gran criterio que podría ser aducido como nuevo cleavage en el mercado de trabajo sea la carencia de una cualificación mínima, que condenaría al trabajador a quedar atrapado en un círculo vicioso de precariedad, temporalidad, escasa retribución, esporádico desempleo, etc.

8. Una síntesis de esta cuestión puede encontrarse en R. Freeman (1994), Working under different rules. 
Se produjo un descomunal aumento de la desigualdad salarial y un gran deterioro en los ingresos de los trabajadores peor pagados.

Tomando como trasfondo estas experiencias divergentes (aumento de la desigualdad en EEUU y aumento del desempleo en Europa), trataremos de analizar qué papel ha podido jugar la mano de obra inmigrante en uno y otro caso.

\section{EEUU. Evidencia empírica disponible}

Hablar de un vínculo entre el aumento de la desigualdad salarial y la inmigración tiene sentido en la medida en que ésta puede modificar la oferta de trabajo en un país, no sólo cuantitativa sino también cualitativamente. Para ser más exactos, si las modificaciones en la demanda de mano de obra desde los setenta actuaron en favor de los trabajadores con mayores niveles de cualificación ${ }^{9}$ y el perfil dominante entre los trabajadores inmigrantes (especialmente de los que entraron a partir de esa fecha) es el de un trabajador con poca cualificación, es evidente que la inmigración puede actuar en contra del segmento de trabajadores nativos menos cualificados, en la medida que trabajadores con el mismo tipo de características laborales, con independencia de su nacionalidad, sean sustituibles en el proceso productivo. Nótese que estamos añadiendo un matiz importantísimo al modelo de análisis inicialmente formulado por la escuela neoclásica y que no debe pasar inadvertido: concentrar la atención en la repercusión que la inmigración puede tener sobre un grupo específico de trabajadores nativos, los menos cualificados.

Greenwood y McDowell, en su revisión de la literatura de 1986, señalaban la escasez de estudios empíricos sobre la cuestión debido a la inexistencia de los datos requeridos. Desde entonces, la aparición de fuentes adecuadas ha permitido numerosas investigaciones, sobre todo de carácter local. Inicialmente, en la década de los ochenta, tales estudios llegaron a conclusiones sorprendentemente similares: el efecto de la mano de obra inmigrante sobre el nivel de empleo y los salarios de distintas categorías de trabajadores nativos (desagregados por raza, sexo, edad) es prácticamente inapreciable ${ }^{10}$. En cambio, las investigaciones desarrolladas en los noventa, en gran medida a partir de las críticas realizadas a los análisis de ámbito local, han cuestionado el consenso reinante hasta entonces en la materia, dando apoyo empírico a la idea neoclási-

9. Los cambios en la demanda, motivados por la internacionalización de la economía americana y por el cambio tecnológico, podrían ser alegados como causa fundamental del aumento del diferencial salarial entre trabajadores más y menos cualificados. Sin embargo, teniendo en cuenta $a$ ) que dichas transformaciones ya venían produciéndose en EEUU en los setenta sin que se observara un aumento de la desigualdad y b) que en los países europeos avanzados, idénticas transformaciones en la demanda tampoco generaron tal desigualdad, parece razonable pensar que la explicación debe ir más por el lado de la oferta y/o de los elementos institucionales.

10. Entre los estudios realizados en la década de los ochenta podemos citar, por orden de aparición, los siguientes: Grossman, 1982; Borjas, 1983, 1984; De Freitas, 1988; LaLonde y Topel, 1991; Altonji y Card, 1991. 
ca de que los inmigrantes compiten, al menos, con algunos trabajadores nativos en los mercados de trabajo locales a los que se incorporan.

El primer grupo de estudios empíricos comparaban el empleo y/o los salarios de trabajadores nativos en ciudades con una elevada proporción de trabajadores inmigrantes, con los salarios de trabajadores nativos en ciudades donde la oferta de mano de obra inmigrante era mínima, controlando por otras variables que influyen sobre la productividad y el nivel de retribución regional (tasa de desempleo, coste de la vida, productividad del factor trabajo, densidad sindical, etc.). La conclusión solía ser la misma: la correlación entre la proporción de mano de obra nacida en el extranjero y el nivel de los salarios nativos es bastante débil, aunque generalmente negativa. Por tanto, estos primeros estudios corroboraron, en términos generales, la tesis de Piore sobre la no-concurrencia de mano de obra inmigrante y nativa debido a su concentración en distintos segmentos del mercado de trabajo.

Ante la escasa importancia de los efectos observados, era frecuente realizar proyecciones y establecer "umbrales de tolerancia», es decir, la proporción de mano de obra inmigrante por encima de la cual ligeros incrementos adicionales en la misma provocarían una reducción sustancial en los salarios de los nati$\operatorname{vos}^{11}$. Tales proyecciones y los consiguientes umbrales deben contemplarse con suma cautela y ser utilizados con fines meramente ilustrativos por diversos motivos. En primer lugar, estas proyecciones se hacen tomando como escenario de referencia el definido para el momento actual y no contemplan la posibilidad de que, mientras se alcanzan dichos umbrales (que suelen estar aún muy lejos de las proporciones de población inmigrante actuales), se produzcan cambios importantes en la estructura productiva y en la cualificación relativa de la mano de obra nativa e inmigrante ${ }^{12}$. Es decir, implícitamente se conciben los mercados de trabajo locales como mercados cerrados tras la incorporación de los inmigrantes y, en consecuencia, no se valora la posibilidad de que los trabajadores nativos modifiquen su comportamiento laboral en respuesta a la llegada de esa nueva mano de obra, ni que ésta favorezca inversiones en otros sectores, ni que reformas institucionales, por ejemplo relativas al subsidio de desempleo o al salario mínimo, de gran incidencia sobre el segmento de la población trabajadora que nos interesa (trabajadores de escasa cualificación y bajos salarios), modifiquen las esperadas relaciones de sustitución entre trabajadores.

11. De Freitas y Marshall, por ejemplo, señalan que el efecto negativo sólo empezaría a ser considerable cuando la proporción de trabajadores inmigrantes superase el $20 \%$ de la mano de obra total en una industria determinada; a partir de dicho umbral, un $1 \%$ adicional de mano de obra inmigrante provocaría una reducción del 1,2\% en la tasa de crecimiento de los salarios (De Freitas y Marshall, 1984).

12. Es de esperar que el nivel de cualificación de los trabajadores extranjeros varíe con los años de residencia en el país de acogida. Además, cabe la posibilidad de que, en el futuro, la composición interna de la población inmigrante admitida en un determinado país se modifique, pues tanto las políticas de inmigración como las transformaciones en los países de salida juegan un papel crucial en la definición del perfil sociodemográfico de los inmigrantes (Bauer et al., 2000). 
Las investigaciones de los noventa representan un giro importante, pues desacreditan en cierta medida las conclusiones de no-competición o no-concurrencia entre trabajadores inmigrantes y nativos. Dicho giro se explica fundamentalmente por dos motivos:

- En primer lugar, empieza a tomarse en cuenta el nivel de cualificación relativo de inmigrantes y nativos. Altonji y Card (1991), por ejemplo, demuestran cómo el impacto de la inmigración sobre los salarios puede depender de la distribución de cualificaciones de los inmigrantes en relación con la de los nativos ${ }^{13}$.

- En segundo lugar, diversos estudios empiezan a introducir en sus estimaciones elementos que permiten superar la asunción de que los mercados locales a los que se incorporan los inmigrantes son mercados cerrados: unos, como Borjas, Freeman y Katz (1992), incluyen en el análisis el efecto de la circulación de bienes y servicios; otros, analizan la movilidad de la propia mano de obra nativa tras la llegada de inmigrantes.

En el trabajo de Borjas y otros, tras calcular cuál ha sido la influencia del comercio y la inmigración sobre los cambios en la distribución de cualificaciones de la mano de obra (la oferta relativa de mano de obra menos cualificada - menos de doce años de estudios — respecto a la más cualificada), se traducen estos cambios en efectos sobre el diferencial de salarios entre ambos grupos. Y la conclusión alcanzada difiere ampliamente de las de los estudios de ámbito local de los años ochenta: ahora los cambios en la cualificación de la fuerza de trabajo inducida conjuntamente por el comercio y la inmigración explicaría algo más del $40 \%$ del declive relativo experimentado por los salarios de los trabajadores con menos de doce años de estudios, respecto a los que tienen doce años o más, entre 1980-1988 ${ }^{14}$. Este efecto es sustancial comparado con el atribuido por otros estudios a factores como cambios en la composición sectorial de la industria o el declive de la densidad sindical.

El segundo grupo de estudios señalan cómo, ante la llegada de inmigrantes, muchos trabajadores nativos emigran hacia otras localidades menos afectadas por la inmigración, eliminando así el impacto previsible del aumento de mano de obra sobre el empleo y los salarios. En lugar de cambiar los salarios,

13. Concluyen que tanto los salarios de los trabajadores cualificados como de los no cualificados permanecen invariables cuando la proporción de trabajadores no cualificados en el flujo de inmigrantes es igual a la proporción de trabajadores no cualificados en la población nativa; sin embargo, si la proporción de no cualificados entre el contingente de inmigrantes es mayor que la proporción respectiva entre la población nativa, el efecto es un aumento de los salarios de los cualificados, pero un descenso de los salarios de los no cualificados.

14. Es importante resaltar que, en dicho trabajo, los autores no separan el efecto atribuible al comercio y a la inmigración. Sin embargo, Borjas, en su revisión de la literatura de 1994, se refiere explícitamente a este trabajo y destaca como una de sus conclusiones que "quizás un tercio de los diez puntos porcentuales que ha bajado el salario relativo de los trabajadores con menos de doce años de estudio (high school dropouts) entre los años 1980 y 1988 puede ser atribuida al flujo de inmigración menos cualificada» (Borjas, 1994: 1699). 
es la composición de la mano de obra lo que cambia. En pocas palabras, la movilidad de los nativos disipa el impacto de la inmigración ${ }^{15}$.

\section{Europa. Evidencia empírica disponible}

Como ya indicamos, la investigación sobre el impacto económico de la inmigración en Europa es mucho más fragmentaria, parcial e inconclusa que en EEUU. Como era de esperar, los estudios más serios sobre la cuestión se han realizado para aquellos países en los que el reclutamiento de mano de obra extranjera tuvo una importancia significativa hasta los años setenta (Suiza y Alemania, sobre todo), tratando a menudo de compararse su repercusión económica hasta el cierre de fronteras en 1974 y con posterioridad a esta fecha ${ }^{16}$.

Burgemmeier (1992) se pregunta sobre el grado de complementariedad o sustitución entre los diferentes factores de producción en la economía suiza y concluye que el mayor grado de complementariedad se da entre capital y mano de obra extranjera, mientras que los dos factores más sustituibles en el proceso de producción son el capital y la mano de obra nativa. La explicación ofrecida es, como es lógico, la diferente distribución de cualificaciones entre ambos sectores de la mano de obra y, aunque no encuentra consecuencias negativas sobre los salarios de los nativos, acaba proponiendo una limitación del reclutamiento de mano de obra extranjera que utilice el nivel de cualificación como criterio de selección ${ }^{17}$.

Para el caso alemán, Zimmermann y otros, usando datos del West German Socio-Economic Panel, concluyen que en los años setenta la considerable proporción de mano de obra extranjera aumentó la frecuencia del desempleo entre

15. Filer, 1992; White y Hunter, 1993; Frey, 1994.

16. Para una revisión de los estudios empíricos realizados en España, véase Carrasco, 1999: 45-51. Se trata casi únicamente de estudios de carácter local y sectorial. Sólo conocemos una estimación del impacto de la inmigración sobre los salarios y el empleo nativo realizada a escala nacional, tras la regularización de 1991, por Dolado y otros (1996). Dicho trabajo concluye que hay efectos umbral, de modo que para bajas tasas de inmigración puede ocurrir que la situación de los no cualificados en el mercado de trabajo mejore. Concretamente se destaca que la situación laboral de los nativos no cualificados no empeoró tras el proceso de regularización de 1991: el efecto sobre los salarios es pequeño pero positivo y el efecto sobre el empleo es negativo pero no significativo.

17. Tal propuesta es consecuencia de una serie de razonamientos encadenados que el autor realiza en base a los datos: a partir de 1965 se observa una disminución en la tasa de acumulación de capital, coincidiendo con la reducción de mano de obra extranjera, sin embargo esto sólo se traduce lenta y parcialmente en una reducción de la tasa de crecimiento del PNB. Ello se debe, según el autor, no sólo a la necesidad de que transcurra un mayor lapso de tiempo para que las consecuencias puedan apreciarse, sino fundamentalmente, y ésta es la hipótesis central, a que los empresarios se quedan con los trabajadores más productivos. Es decir, se baraja la hipótesis de que, desde 1965, se logra mantener la tasa de crecimiento económico con menor cantidad de mano de obra inmigrante, debido a que se produce un incremento en la productividad marginal de los trabajadores extranjeros; de ahí la sugerencia de usar el nivel de cualificación como criterio selectivo. 
los alemanes, mientras que en los ochenta (usando datos de 1984-1989) el efecto negativo se produce sobre los salarios. Al examinar los salarios, este trabajo sí que diferencia a los trabajadores según su cualificación (manuales y de cuello blanco), con la intención de contrastar la hipótesis de partida (que la inmigración reducirá el salario de los poco cualificados pero aumentará el de los cualificados), basada en las previsibles diferencias en el nivel de cualificación (medio) entre nativos e inmigrantes. Los resultados, controlando en los modelos por variables tanto de orden individual (años de escuela, años de experiencia, meses desempleado en los últimos años, estado civil) como de tipo más estructural (tipo de sector de actividad, crecimiento del sector, tamaño de la empresa, tasa regional de desempleo), corroboran dicha hipótesis: 1) en el conjunto de los sectores analizados, el coeficiente de la variable «proporción de mano de obra extranjera» es siempre negativo y significativo ${ }^{18}$; 2) distinguiendo el efecto de la mano de obra inmigrante sobre los salarios de trabajadores manuales y no manuales, para comprobar si puede hablarse de un trade-off entre ambos tipos (que el efecto para unos sea positivo y para otros, negativo), se estima que el efecto negativo sobre los salarios de los trabajadores manuales $(-5,9 \%)$ supera el efecto positivo sobre los de cuello blanco $(3,3 \%)$ y, por tanto, se concluye que el efecto global de la inmigración sobre los salarios de los nativos es negativo, es decir que, en términos generales, la mano de obra extranjera y la alemana son sustitutos.

También usando datos del SOEP, de 1984-1991, Hatzious (1995) obtiene resultados muy similares a los de Zimmerman: la inmigración no parece afectar al desempleo nativo sustancialmente y, en cambio, parece que sí que afecta negativamente a los salarios ${ }^{19}$.

Los resultados obtenidos para el caso italiano contrastan ampliamente con los de Alemania. Utilizando datos del Archivo de la Seguridad Social de Empleo Privado (que representa el 56,2\% del empleo total italiano pero que sí recoge el grueso de los inmigrantes legales) para el período 1986-1995, Gavosto y otros (Gavosto, 1999) concluyen que el efecto sobre los salarios de los nativos es pequeño pero positivo (un aumento del $1 \%$ en la proporción de inmigrantes provoca un aumento de los salarios nativos del 0,01\%). Además, este efecto positivo es mayor entre los trabajadores manuales, entre los empleados en pequeñas empresas y entre los empleados en el norte del país. Por tanto, la conclusión es que entre trabajadores italianos manuales y mano de obra inmigrante existe una relación de complementariedad que los autores explican por la inexistencia de trabajadores nativos dispuestos a desarrollar ciertos trabajos.

18. Concretamente se estima que un incremento del $1 \%$ en la proporción de inmigrantes provocaría una reducción de los salarios de un $0,35 \%$. Así que si se pasara del 8,5\% (proporción media de inmigrantes en el conjunto de los sectores) al 9,5\% - lo que equivale a un incremento de la proporción de inmigrantes del $11,7 \%$ — se produciría una reducción salarial del $4,1 \%$.

19. Según las regresiones realizadas, por cada $1 \%$ que crece la población activa debido al influjo de inmigrantes, los salarios nativos descienden en torno a un $7 \%$. 
Ahora bien, si la razón del efecto positivo es que los inmigrantes rellenan huecos a bajo coste, es posible que, a medida que aumente la cantidad de inmigrantes, no exista suficiente demanda para ese tipo de empleos, y que el efecto positivo vaya desapareciendo. De hecho, los autores señalan la existencia de un umbral máximo (entre el 7 y el 12\%), a partir del cual un aumento en la proporción de inmigrantes los convertirá en un factor competitivo para los trabajadores nativos ${ }^{20}$.

Sorprende lo opuesto de los resultados obtenidos para cada uno de estos dos últimos países: en Alemania los inmigrantes aparecen como sustitutos de los trabajadores alemanes, más concretamente de los manuales; además el estudio de Zimmermann señalaba que los casados, los que trabajan en grandes ciudades y en grandes empresas, obtenían mejores salarios. En cambio, para el caso italiano, la conclusión es que los inmigrantes son complementarios de los trabajadores italianos, en especial de los manuales, más aún si trabajan en el norte del país y en pequeñas empresas.

Lo anterior induce a pensar que: $a$ ) la diferente secuencia temporal del fenómeno inmigratorio en los distintos estados miembros de la Unión Europea, y b) las diferencias entre sus mercados de trabajo, tienen consecuencias inmediatas sobre la situación laboral de la mano de obra extranjera en cada uno de estos estados y, por tanto, también, sobre su repercusión en el conjunto de las economías nacionales de los países de acogida. Por tanto, antes de diseñar una política de inmigración para toda la UE, parece innegable la necesidad de que haya un conocimiento en profundidad de tales diferencias que permita un diseño adecuado de la política de inmigración común, que no tiene por qué significar única.

\section{Conclusiones}

Conocida la evidencia empírica disponible, a la pregunta inicial $-\prec H a$ influido la inmigración en el aumento de la desigualdad en EEUU y del desempleo en Europa? - podemos responder que:

\section{La inmigración no ha fomentado el desempleo en ninguno de los dos conti- nentes.}

Esto era más o menos previsible para el caso norteamericano, pues el menor grado de protección salarial debía permitir un reajuste total del mercado laboral, ante el aumento de mano de obra no cualificada, vía precio (es decir salario). En cambio, la predicada rigidez salarial de los mercados de trabajo europeos pronosticaba, conforme a la teoría económica más clásica, un desplazamiento de trabajadores nativos en el segmento menos cualificado, al no poderse compensar el incremento de la oferta laboral con un descenso del salario de equilibrio. Sin embargo, dicho desplazamiento no se aprecia en ninguno de los estudios empíricos revisados.

20. En cualquier caso, recuérdense aquí las advertencias que para el caso de EEUU hicimos sobre la cautela con que deben tomarse este tipo de proyecciones. 
Ante dichos resultados para el caso europeo caben dos explicaciones: a) los trabajadores inmigrantes simplemente ocupan puestos de trabajo que, con independencia de su grado de cualificación, los trabajadores nativos rechazan, siguiendo la formulación más radical de la hipótesis de la segmentación defendida por Piore $o, b$ ) en la práctica, sí que existe cierta flexibilidad salarial en los mercados de trabajo europeos, al menos en los sectores en los que suelen integrarse los trabajadores inmigrantes ${ }^{21}$.

\section{El efecto de la mano de obra inmigrante sobre los niveles salariales sigue resul- tando ambiguo.}

La mayoría de las investigaciones realizadas hasta principios de los años noventa indicaban un efecto negativo sobre los salarios mayor en Europa que en EEUU. Numerosos factores pueden ayudar a explicar este hecho. Por una parte, en Europa existe mayor discontinuidad salarial ${ }^{22}$ y mayor concentración de los inmigrantes en determinadas ocupaciones o sectores productivos que en EEUU ${ }^{23}$. Por otra, EEUU ha favorecido el carácter permanente de la inmigración, mientras que en Europa se ha fomentado la inmigración de carácter temporal que se caracteriza, como muestran los estudios de Dustman, por presentar mayores tasas de actividad y menores salarios de reserva (Dustman, 1993).

Además, la mayor movilidad (geográfica) de la mano de obra norteamericana, frente a la europea, puede hacer que el potencial efecto negativo de la inmigración sobre los salarios se disipe. Es decir que, aun existiendo dicho impacto negativo, el tipo de estudios realizados hasta hace poco puede que no fuesen apropiados para apreciarlo. De hecho, los últimos estudios aparecidos en EEUU, que ya utilizan series temporales y datos a nivel nacional, atribu-

21. En este sentido, Gehrig y otros plantean la idea de que la potencial reducción en el nivel de empleo de los nativos poco cualificados que supondría la llegada de trabajadores inmigrantes, podría incentivar una flexibilización de la postura sindical respecto al nivel de salarios, induciendo al sindicato a admitir salarios algo menores, a sabiendas de que ello permitiría incrementar el empleo total en el segmento de los pocos cualificados, compensando en algo las pérdidas de ganancia salarial derivada de la reducción en el nivel de salarios (Gehrig, 1992).

22. En palabras de Livi-Bacci, en EEUU «hay muy poca diferencia entre un inmigrado ilegal y un trabajador legal. Un trabajador ilegal mejicano que cruza la frontera de Río Grande y se va a trabajar a un fast-food de Los Angeles, gana un sueldo de 5 dólares a la hora y el estudiante americano, nacido en Los Angeles, que va a trabajar a McDonald's, cerca del otro, gana 5 dólares con 25 o 50 centavos. La diferencia es mínima y hay continuidad en la progresión salarial. En Europa no. Hay una diferencia entre el sueldo de un inmigrado marginado, que no consigue la entrada en el mercado oficial de trabajo, y el autóctono, que está en un mercado de trabajo protegido y estructurado. Hay una diferencia de uno a dos, o de uno a tres, o más; por tanto no hay esta continuidad» (LiviBacci, 1991: 28-29).

23. Zimmermann muestra cómo en EEUU el trabajo inmigrante sigue, en líneas generales, las pautas de concentración ocupacional del empleo total (salvo para el caso de la industria), mientras que en Europa existe una mayor diferenciación ocupacional entre nativos e inmigrantes (Zimmermann, 1995: 254-256). 
yen un peso importante a la inmigración como causa del descenso salarial en los últimos escalones de cualificación ${ }^{24}$ y, por tanto, del incremento de la desigualdad en EEUU durante los años ochenta ${ }^{25}$.

En el caso de que futuros estudios confirmen esta responsabilidad de la inmigración en el aumento de la desigualdad, los defensores de políticas restrictivas encontrarían el apoyo "estadístico» que tanto tiempo llevan esperando. Sin embargo, la contrarréplica es bien sencilla: una de las mayores dificultades con las que se encuentra la política de inmigración es, efectivamente, que su impacto sobre la fuerza de trabajo puede implicar perjuicios para grupos concretos de forma casi inmediata. Ahora bien, como en el caso de las medidas de apertura comercial, no estamos tratando con un juego de suma cero, en el que la economía receptora pierde todo lo que los inmigrantes ganan, sino ante un problema de reparto de los beneficios y perjuicios que derivan de la contratación de mano de obra inmigrante. Es decir, estamos ante una cuestión redistributiva y, por tanto, eminentemente política. Así pues, más que para propugnar la adopción de políticas que protejan a toda costa a los potenciales perjudicados de cualquier competencia en sus actuales empleos, estos estudios deberían servir para prevenir, estimulando la reflexión sobre qué medidas podrían amortiguar transitoriamente los efectos de la inmigración sobre los grupos potencialmente perjudicados, favoreciendo su capacidad de ajuste a nuevas ocupaciones, en beneficio propio y del conjunto de la sociedad.

\section{Bibliografía}

AltonjI, J.; CARD, D. (1911). «The effects of immigration on the labor market outcomes of less-skilled natives». En ABOWD, J.M.; FREEMAN, Richard B. Immigration, Trade, and the Labor Market. Chicago: University of Chicago Press.

ABrams, E.; ABrams, F.S. (1975). «Immigration policy. Who gets in and why?». The Public Interest, 38, p. 3-29, invierno.

Ahn, N.; De la Rica, S.; Ugidos, A. (1998). «Willingness to Move for Work and Unemployment Duration in Spain». CEMFI, Working Paper 9801, abril.

Baganha, M.I.; FerraO, J.; Macaísta, J. (1999). «Os imigrantes e o mercado de trabalho. O caso português». Analise Social XXXIV, núm. 150, p. 147-73.

BALDACCI, E.; INGLESE, L.; STROZZA, S. (1999). «Determinants of Foreign Workers' Wages in Two Italian Regions with High Illegal Immigration». Labour, 13, núm. 3, p. 675-710.

24. Borjas, Freeman y Katz, 1992; Camarota, 1998.

25. De todos modos, tales resultados son aún "preliminares» y adolecen de un serio problema metodológico, denominado «efecto composición», que contribuye a sobreestimar los efectos de la inmigración sobre el descenso salarial sufrido por los nativos menos cualificados en los últimos años. El «efecto composición» hace referencia a que el nivel salarial de una ciudad, región o país, es un compuesto de los salarios de nativos y de inmigrantes de dicho lugar; de modo que si los inmigrantes ganan menos que los nativos (como suele ocurrir), las ciudades o países con mayores proporciones de inmigrantes tendrán salarios medios menores, incluso aunque los inmigrantes no tuvieran ningún impacto negativo sobre los salarios nativos. 
Bauer, T.; Lofstrom, M.; Zimmermann, K.F. (2000). «Immigration policy, assimilation of immigrants and natives' sentiments towards immigrants: evidence from 12 OECD-countries». Discussion Paper, núm. 187, IZA, agosto.

BORJAS, G.J. (1983). "The substitutability of Black, Hispanics and White labor». Economic Inquiry, 21, p. 93-106.

- (1984). "The impact of immigrants on the earnings of the native-born». Nueva York: Editorial Basic Books.

- (1989). "Economic Theory and International Migration». International Migration Review, XXIII, núm. 3, p. 457-85.

- (1990). Friends or Strangers: The impact of immigrants on the US Economy. Nueva York: Basic Books.

- (1994). "The economics of immigration». Journal of Economic Literature, XXXII, p. 1667-717, diciembre.

BorJas, G.J.; FreEMAN, Richard B. (1992). Immigration and the Work Force: Economic Consequences for the United States and Source Areas. Chicago: University of Chicago Press.

Borjas, G.J.; TiEndA, M. (1987). «The Economic Consequences of Immigration». Sciences, 235, p. 645-51, 6 de febrero.

- "The Employment and Wages of Legalized Immigrants». International Migration Review, XXVII, núm. 4, p. 712-47.

Bover, O.; Arellano, M.; Bentotlila, S. (1997). «Unemployment Duration, Benefit Duration and the Business Cycle». CEMFI, Working Paper 9717, octubre.

BurgenMeIER, B. (1992). Main d'oeuvre étranger. Une analyse de l'economie suisse. Paris: Economica.

BUSSERY, Henry (1976). «Incidence sur l'économie française d'une réduction durable de la main d'oeuvre immigré». Economie et Statistique, 76, p. 37-45.

Camarota, Steven A. (1998). The wages of immigration. The effect on the low-skilled labour market. Washington: Center for Immigration Studies.

Carrasco Carpio, Concepción (1999). Mercados de trabajo: los inmigrantes económicos. Madrid: Instituto de Migraciones y Servicios Sociales. Colección Observatorio Permanente de la Inmigración.

Chiswick, B.R.; ChIswick, C.U.; Miller, P.W. (1986). «Are Immigrants and Natives Perfect Sustitutes in Production?». International Migration Review, XIX, núm. 4, p. 674-84.

Cohen, A. (1995). "Algunas reflexiones a propósito de la inmigración magrebí en España». Ería, 38, p. 287-302.

COLECTIVO IOÉ (1998). Inmigración y trabajo: trabajadores inmigrantes en el sector de la construcción. Madrid: OPI, IMSERSO.

Coleman, J. (1999). "New light in Mediterranean Migration». Internationa Migration, vol. 37 (2), p. 485-513.

Cornelius, W.A.; Martin, P.L.; Hollifield, J.F. (eds.) (1994). Controlling immigration. A global perspective. California: Stanford University Press.

De Freitas, G.; MARShall, A. (1984). "Immigration and wage growth in US manufacturing in the 1970s». En DENnIS, Barbara D. (ed.). Proceedings of the XXXVI Annual Meeting. IRRA, p. 148-56. 
- (1988). «Hispanic immigration and labor market segmentation». Industrial Relations, 27 (2), p. 195-214.

De New, J.P.; Zimmermann, K.F. (1994). "Native Wage Impacts of Foreign Labor: A Random Effects Panel Analysis». Journal of Population Economics, 7, p. 177-192.

DíEz Nicolás, J. (1999). Los españoles y la inmigración. Madrid: Instituto de Migraciones y Servicios Sociales. Colección Observatorio Permanente de la Inmigración.

Dolado, J.J.; Jimeno, J.F.; DuCE, R. (1996). The Effects of Migration on the Relative Demand of Skilled Versus Unskilled Labour: Evidence From Spain. Documento de trabajo, núm. 96-20. FEDEA.

Dustmann, C. (1993). «Earnings adjustment of temporary migrants». Journal of Population Economics, 6, 2, p. 153-168.

FILER, R.K. (1992). "The effect of immigrants arrivals on migratory patterns of native workers». En BORJAS, G.J.; FREEMAN, Richard B. Immigration and the Work Force: Economic Consequences for the United States and Source Areas. Chicago: University of Chicago Press.

FreEMAN, Richard B. (1994). Working under Different Rules. Nueva York: Russell Sage Foundation.

FreY, W.H. (1994). «The new white flight». American Demographics, p. 40-48, abril. FrIEDBERG, Rachel M.; HUNT, Jennifer (1995). «The impact of immigrants on host country wages, employment and growth». Journal of Economic Perspectives, vol. 9, 2, p. 23-44.

Gehrig, A.; SCHMidT, C.M.; ZimmermanN, K.F. (1992). «Mass migration, unions and fiscal migration policy». CEPR Discusion Paper, núm. 727. Londres.

Gavosto, A.; Venturini, A.; Villosio, C. (1999). «Do Immigrants Compete with Natives?». Labour, 13, núm. 3, p. 603-22.

GreEnwood, M.J.; MCDowell, J.M. (1994). «The National Market Consequences of U.S. Immigration». En GIERSCH, H. (ed.). Economic aspects of international migration. Springer-Verlag.

- «The Factor Market Consequences of U.S. Immigration». Journal of Economic Literature, XXIV, p. 1738-72, diciembre.

Grossman, J.B. (1990). «The substitutability of natives and immigrants in production». Review of Economics and Statistics, 64, 4, p. 596-603.

Hollifield, James F. (1992). Immigrants, Markets, and States: The Political Economy of Postwar Europe. Cambridge, Mass: Harvard University Press.

IZQUIERDO, Antonio (1996). La inmigración inesperada. La población extranjera en España, 1991-1995. Madrid, Trotta.

LALONDE, R.; TOPEL, R. (1991). "Labor market adjustments to increased immigration». En ABOWD, J.M.; FREEMAN, Richard B. Immigration, Trade, and the Labor Market. Chicago: University of Chicago Press.

LIVI-BACCI, M. (1991). Inmigración y desarrollo: comparación entre Europa y América. Barcelona: Fundación Paulino Torras Domenech.

MarTíneZ Veiga, U.; SANTANA Afonso, A. (1997). «Inmigración y mercado laboral». En PUYOL, Antolín et al. (eds.) Cuadernos de Relaciones Laborales, núm. 10. 
OCDE (1993). Tendances Des Migrations Internationales. Rappot Annuel. París.

PIORE, M.J. (1979). Birds of passage: migrant labour and industrial societies. Cambridge: Cambridge University Press.

SCHMidT, C.M.; STILZ, A.; ZimmermanN, K.F. (1994). «Mass Migration, Unions and Government Intervention». Journal of Public Economics, 55, p 185-201.

SimON, Julian L. (1999). The economic consequences of immigration. University of Michigan Press, $2^{\mathrm{a}}$ ed.

Varios Autores (1998). South Europe Society and Politics, núm. 3. Arango, M.; BALDWIN-EDWARDS, J. (eds.). Madrid: Frank Cass Journal, invierno.

WHITE, M.J.; HUNTER, L. (1993). The migratory response of native-born workers to the presence of immigrants in the labor market. Brown University, julio.

ZiMMMERMANN, K.F. (1994). «Some general lessons for Europe's migration problem». En GIERSCH, H. (ed.). Economic aspects of international migration. Springer-Verlag. 